Sādhanā Vol. 38, Part 6, December 2013, pp. 1259-1285. (C) Indian Academy of Sciences

\title{
Modified enthalpy method for the simulation of melting and solidification
}

\author{
NIRANJAN N GUDIBANDE* and KANNAN N IYER \\ Department of Mechanical Engineering, Indian Institute of Technology Bombay, \\ Mumbai 400 076, India \\ e-mail: niranjangn@iitb.ac.in; kiyer@iitb.ac.in
}

MS received 18 February 2012; revised 19 February 2013; accepted 6 March 2013

\begin{abstract}
Enthalpy method is commonly used in the simulation of melting and solidification owing to its ease of implementation. It however has a few shortcomings. When it is used to simulate melting/solidification on a coarse grid, the temperature time history of a point close to the interface shows waviness. While simulating melting with natural convection, in order to impose no-slip and impermeability boundary conditions, momentum sink terms are used with some arbitrary constants called mushy zone constants. The values of these are very large and have no physical basis. Further, the chosen values affect the predictions and hence have to be tuned for satisfactory comparison with experimental data. To overcome these deficiencies, a new cell splitting method under the framework of the enthalpy method has been proposed. This method does not produce waviness nor requires mushy zone constants for simulating melting with natural convection. The method is then demonstrated for a simple onedimensional melting problem and the results are compared with analytical solutions. The method is then demonstrated to work in two-dimensions and comparisons are shown with analytical solutions for problems with planar and curvilinear interfaces. To further benchmark the present method, simulations are performed for melting in a rectangular cavity with natural convection in the liquid melt. The solid-liquid interface obtained is compared satisfactorily with the experimental results available in literature.
\end{abstract}

Keywords. Melting; enthalpy method; wavy interface; mushy zone constant

\section{Introduction}

The study of melting and solidification offers insights in the design of casting, welding, latent thermal energy storage systems, etc., and in the analysis of melting of glaciers, solidification of lava, thawing of food, etc. The motivation for the present work arose from a need to analyse

*For correspondence 
melting of lead in a metallic cask due to fire. Numerical tools are valuable in such a study. Numerical techniques for simulating melting and solidification must be capable of handling the presence of a moving solid-liquid interface (an internal boundary) across which the properties may be discontinuous. They must also be capable of handling natural convection in the molten liquid. Several numerical methods exist for simulating melting/solidification and phase change phenomena in general. These methods can be classified into deforming grid, front tracking and enthalpy methods.

In a deforming grid method, meshes are created for the solid and the liquid domains separately with the solid-liquid interface as a common boundary. Governing equations are solved for temperature and velocity fields with appropriate boundary conditions at the solid-liquid interface. Stefan condition (an energy balance at the solid-liquid interface) is used to obtain the velocity with which the interface moves as the material undergoes melting/solidification. This velocity is integrated in time to obtain the new location of the interface. Deforming grid method requires creation of grids at each time step. This method has been used for the simulation of melting of pure metals with natural convection in the molten liquid by Lacroix (1989), Cerimele et al (2002) and Kumar et al (2006).

In a front tracking method, the interface is represented by a set of marker points connected by curves (in $2 \mathrm{~d}$ ) or triangular elements (in 3d). These marker particles move on a fixed grid as the material undergoes melting or solidification. The governing equations are solved on the fixed grid and for nodal points which are close to the interface finite difference stencils which involve nodal points of the same phase and one or more points on the interface are used. The velocity of the marker points are obtained by applying the Stefan condition. As the material undergoes melting or solidification, these marker points may form clusters or move far apart and thus redistribution of the marker particles becomes necessary. Udaykumar et al (1996) and Li et al (2003) have demonstrated this method for melting and solidification. Both the above mentioned methods require special treatment when the interface undergoes a topological change due to merging or splitting of interface.

In the enthalpy method, the energy equations of the solid and the liquid domain and the boundary conditions on the interface are combined into a single equation called the enthalpy equation. By solving the enthalpy equation the temperature and the liquid fraction fields $(f)$ can be obtained. Liquid fraction is the ratio of volume of liquid in a control volume (CV) to the total volume of the CV. Thus the solid and the liquid control volumes have $f=1$ and $f=0$, respectively and control volumes which have $f \in(0,1)$ contain the solid-liquid interface. The enthalpy method is both simple and robust. It is simple in the sense that no complicated algorithms are required for recreation of grids (as in deforming grid method) and in finding suitable stencils for finite difference approximation (as in front tracking method). It is also robust as it does not require any special treatment when the interface undergoes topological changes. This method has been used by several researchers such as Gartling (1980), Voller et al (1987), Brent et al (1988), Cao \& Faghri (1990).

The enthalpy method however has some shortcomings. When enthalpy method is used to simulate melting/solidification on a coarse grid and the temperature of a point close to the interface is plotted as a function of time, it shows a wavy character. This has been attributed to the fact that the temperature of the control volume containing the interface is imposed to be at the melting temperature rather than at the actual location of the solid-liquid interface. Since the discovery of this phenomena by Price \& Slack (1954) several methods have been proposed in the past to eliminate the waviness. These include the implicit time stepping method of Voller \& Cross (1981), explicit enthalpy method of Tacke (1985), centroidal temperature correction method of Date (1994) and the effective conduction length method of Kim \& Anghaie (2001). The 
implicit time stepping and the effective conduction length model are limited for one-dimensional problems. In the centroidal temperature correction method the interface becomes diffuse.

In simulating melting with natural convection, the impermeability and the no-slip condition has to be imposed at the solid-liquid interface. Since the exact location of the solid-liquid interface is unknown in the enthalpy method, these boundary conditions are enforced indirectly. Authors such as Voller et al (1987), Brent et al (1988) have used momentum sink terms in the momentum equations, while Gartling (1980) and Cao \& Faghri (1990) have used a variable viscosity method. In variable viscosity method, viscosity is written as a function of liquid fraction. This function ensures that the viscosity of the solid is very large and approaches that of the molten liquid when $f=1$.

The sink terms and variable viscosity models use large numbers varying from $10^{5}-10^{7}$ as constants (termed mushy constants). Very few studies are available on the sensitivity of the solution to these constants. In a recent study by Shmueli et al (2010), the effect of these mushy constants on the solution was examined. The authors concluded that there exists an optimum value for the mushy constant which would match the experimental results well. Thus, there exists some arbitrariness in specifying these constants. To overcome these deficiencies, Niranjan \& Iyer (2011) had recently proposed a cell splitting method. This paper provides complete details of the cell splitting method.

\section{The governing equation}

The continuity, momentum and the energy equations can be used to obtain the temperature and velocity fields in the liquid and the energy equation can be used to obtain the temperature field in the solid. The molten liquid is assumed to be incompressible and Boussinesq approximation for natural convection is assumed to be valid. The integral forms of the governing equations along with the appropriate boundary conditions at the solid-liquid interface are listed below.

\section{Continuity equation}

$$
\int_{A} \mathbf{V} \cdot \hat{n} d A=0
$$

\section{Momentum equation}

$$
\int_{V} \frac{\partial}{\partial t}(\rho \mathbf{V}) d V+\int_{A} \rho \mathbf{V V} \cdot \hat{n} d A=-\int_{A} P . \hat{n} d A+\int_{A} \mu(\nabla \mathbf{V}) \cdot \hat{n} d A+\int_{V} \rho \mathbf{g} \beta\left(T-T_{r e f}\right) d V .
$$

\section{Energy equations}

In liquid domain

$$
\int_{V} \frac{\partial}{\partial t}\left(\rho C_{P} T\right) d V+\int_{A} \rho C_{P} T \mathbf{V} . \hat{n} d A=\int_{A} k \nabla T . \hat{n} d A
$$

In solid domain

$$
\int_{V} \frac{\partial}{\partial t}\left(\rho C_{P} T\right) d V=\int_{A} k \nabla T \cdot \hat{n} d A
$$


At the solid-liquid interface, the velocity will be zero and the temperature will be set at the melting point. An additional equation can be derived by applying the energy balance at the interface, (known as the Stefan condition)

$$
[-k \nabla T . \hat{n}]_{S}-[-k \nabla T . \hat{n}]_{L}=\rho\left|\mathbf{V}_{\mathrm{n}}\right| \Delta H_{M}
$$

Here, $\mathbf{V}_{\mathrm{n}}$ denotes the velocity of the solid-liquid interface. The direction of this velocity is normal to the interface. The enthalpy method combines the energy equations (3) and (4) along with the boundary conditions into a single equation for enthalpy

$$
\frac{d}{d t} \int_{V} \rho h d V+\int_{A} \rho h \mathbf{V} \cdot \hat{n} d A=\int_{A} k \nabla T . \hat{n} d A .
$$

Here, $h$ denotes the enthalpy. The enthalpy $h$ can be related to the temperature through the following relation,

$$
T-T_{M}= \begin{cases}\frac{h}{C_{P S}} & h<0 \\ 0 & 0<h<\Delta H_{M} \\ \frac{h-\Delta H_{M}}{C_{P L}} & h>\Delta H_{M} .\end{cases}
$$

Here enthalpy of the solid at its melting point is chosen as the reference enthalpy and is assigned a value of zero.

\section{Overall methodology}

In the present method, the control volumes $(\mathrm{CV})$ whose liquid fraction $(f)$ lie in between 0 and 1 are identified. These CVs will henceforth be called as mushy CVs. Using a suitable interface construction method and the $f$ distribution, a piece-wise linear interface is constructed. The constructed interface is used to split the mushy CV into two single phase CVs as shown in figure 1. Thus, if there are $m, n, r$, solid, liquid and mushy $\mathrm{CVs}$, respectively, then the splitting procedure will divide the domain $\Omega$ into liquid sub-domain $\Omega_{L}$ containing $m+r \mathrm{CVs}$ and solid sub-domain $\Omega_{S}$ containing $n+r$ CVs. By solving the mass, momentum and the energy equations in $\Omega_{L}$,

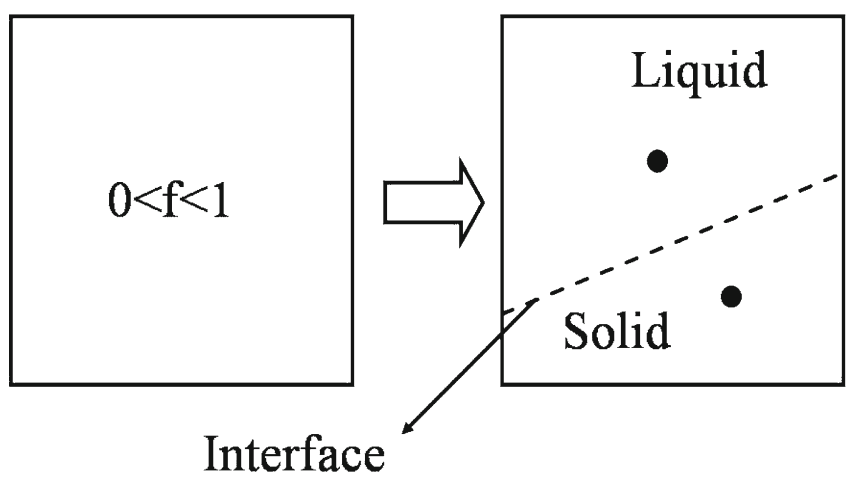

Figure 1. The demonstration of the cell splitting method. 
$u, v$, and $T_{L}$ are obtained, while $T_{S}$ is obtained by solving the energy equation in $\Omega_{S}$. The constructed interface will form a boundary for these equations. For the energy equation, a Dirichlet type of boundary condition is imposed by specifying the interface to be at the melting temperature. The no-slip and the impermeability boundary conditions are imposed for the momentum and the mass equations on the interface. An additional equation is necessary to obtain the evolution of liquid fraction, $f$, with time. This is obtained by solving the enthalpy form of the energy equation for the non-split control volumes.

\section{Interface reconstruction}

Interface reconstruction is an important step in the present method in which an approximate solid-liquid interface is constructed from the liquid fraction data. In volume of fluid method (VOF) too, interface reconstruction plays a vital role. There are several interface construction methods which have been proposed by researchers to simulate two phase flows using VOF methods. A detailed description of several of these methods can be found in Rider \& Kothe (1998) and Pilliod \& Puckett (2004). A typical interface constructed by VOF method, however, produces an interface which is discontinuous at the faces of the control volumes. In VOF method the constructed interface is used to geometrically advect the liquid fractions and the discontinuity does not pose any problem. The present cell splitting method however relies on the existence of a continuous solid-liquid interface and hence a VOF interface reconstruction method cannot be directly used. To produce a continuous piece-wise linear interface the following procedure is employed.

The control volumes sharing a common face and having liquid fractions between 0 and 1 are identified. The solid-liquid interface will pass through the common face of these control volume such as the control volume 'A' and ' $\mathrm{B}$ ' shown in figure 2. The objective is to find the point of intersection of the interface with this common face. It is assumed that the interface can be approximated by a straight line segment in the neighbourhood of the common face. To obtain a unique line representing the solid-liquid interface two constraints have to be imposed. By imposing the conditions that the ratios of volume of liquid to that of the solid in the control volumes $\mathrm{A}$

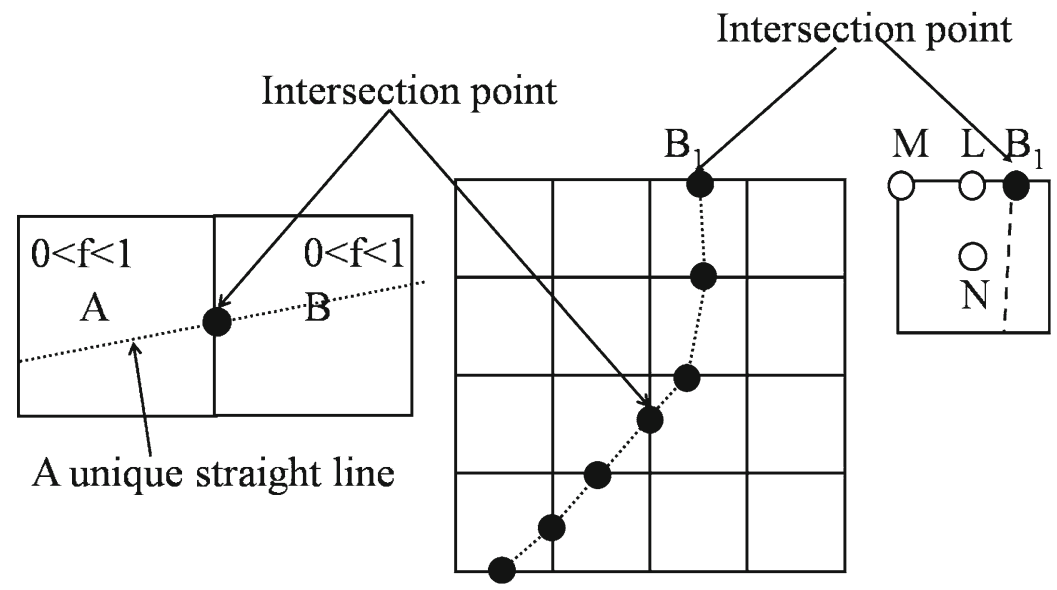

Figure 2. The interface reconstruction method. 
and $\mathrm{B}$ in figure 3 are in the ratios of $f_{\mathrm{A}}: 1-f_{\mathrm{A}}$ and $f_{\mathrm{B}}: 1-f_{\mathrm{B}}$, respectively two constraints can be imposed. Thus a unique straight line can be obtained. This procedure is used for obtaining the intersection points in both horizontal and the vertical directions. The interface is then obtained by joining the intersection points by piece-wise straight lines. Boundary conditions are utilized for finding the boundary intersection point such as point B1 in figure 2. For e.g., if the boundary condition is temperature specified, the temperature gradient in $x$ direction can be obtained from the known temperatures at L and M. Similarly, the gradient in $y$ direction can be obtained from the temperatures at $\mathrm{L}$ and $\mathrm{N}$. Using the two components, the direction of gradient vector can be found. As the interface is an isotherm, its direction will be normal to the gradient vector. Thus, the slope of the interface can be estimated using which a linear interface can be constructed such that it divides the control volume in the ratio of $f: 1-f$. The point of intersection of this constructed interface with the boundary will determine the point B1. Thus, all the points that will constitute an interface are obtained. The line joining these points defines the interface. The procedure followed for the reconstruction is second order accurate and this is derived in Appendix A. The constructed interface is then used to obtain geometric properties such as the centroids, the distances of centroids from the interface, the area of the interface, etc.

The solid-liquid (SL) interface moves as the material undergoes melting or solidification. The SL interface is one of the faces of the split control volume such as the face XY of the control volume ABXYD shown in figure 3. The movement of the face XY results in the expansion/ contraction of the control volume ABXYD. Thus the governing equation has to be modified to account for this movement. Following Donea et al (1982), a generic transport equation for a transport variable $\Phi$ in case of moving grid (interface) can be written as

$$
\frac{d}{d t} \int_{V} \rho \phi d V+\int_{A} \rho \phi\left(\mathbf{V}-\mathbf{V}_{\mathrm{g}}\right) \cdot \hat{n} d A=q_{D}+S,
$$

where $q_{D}$ and $S$ denote the diffusive transport and volumetric source of $\phi$, respectively. The time derivative term outside the integral accounts for the expansion/contraction of the control volume and the $\mathbf{V}_{\mathrm{g}} . \hat{n}$ term ( $\mathbf{V}_{\mathrm{g}}$ is the velocity of the grid) accounts for the convective contribution due to the movement of the grids. The surface area $A$ can be split into the original Cartesian part $A_{\mathrm{C}}$ and the interface part $A_{I}$, for e.g., $A_{\mathrm{C}}$ for the control volume ABXYD in figure 3 is $\mathrm{AB} \cup \mathrm{BX} \cup \mathrm{YD} \cup \mathrm{DA}$ and the interfacial area $A_{I}$ is $\mathrm{XY}$. At the solid-liquid interface due to the
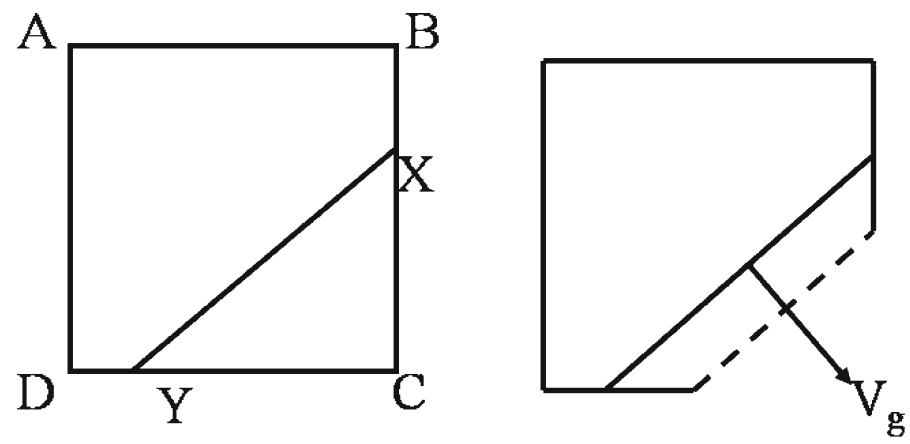

Figure 3. Expansion of control volume due to motion of the solid-liquid interface. 
impermeable boundary condition $\mathbf{V} . \hat{n}$ is zero. Further for the split control volumes the $\mathbf{V}_{\mathrm{g}} . \hat{n}$ term is non-zero only at the solid/liquid interface.

Incorporating the above mentioned simplifications, equation (8) can be written as,

$$
\frac{d}{d t} \int_{V} \rho \phi d V-\int_{A_{I}} \rho \phi \mathbf{V}_{\mathrm{g}} \cdot \hat{n} d A+\int_{A_{\mathrm{C}}} \rho \phi \mathbf{V} \cdot \hat{n} d A=q_{D}+S .
$$

The grid movement should also satisfy the space conservation equation. For a split control volume this can be expressed as,

$$
\frac{d}{d t} \int_{V} d V-\int_{A_{I}} \mathbf{V}_{\mathrm{g}} \cdot \hat{n} d A=0 .
$$

$\phi$ at the solid-liquid interface which represents interface temperature or the velocity components has a constant value determined by the boundary condition. Let $\phi_{I}$ represent this constant value. Multiplying the equation (10) by $\phi_{I}$ and subtracting it from the equation (9) results in

$$
\frac{d}{d t} \int_{V} \rho\left(\phi-\phi_{I}\right) d V+\int_{A_{C}} \rho \phi \mathbf{V} \cdot \hat{n} d A=q_{D}+S .
$$

The temperatures and the velocity fields in the liquid domain and the temperature in the solid domain can be obtained by substituting suitably for $\phi$ in the equation (11). An additional equation is required to obtain the evolution of the interface with time. This is obtained by solving the enthalpy form of the energy equation. The enthalpy equation is solved on the whole control volume such as ABCD in figure 3.

$$
\frac{d}{d t} \int_{V} \rho h d V+\int_{A_{\mathrm{C}}} \rho h \mathbf{V} . \hat{n} d A=\int_{A_{\mathrm{C}}} k \nabla T . \hat{n} d A .
$$

By solving the above equation $\frac{\partial h}{\partial t}$ can be obtained. The enthalpy can be written as a sum of sensible and the latent heat parts, thus

$$
h=f\left[C_{P}\left(T-T_{M}\right)+\Delta H_{M}\right]+(1-f)\left[C_{P}\left(T-T_{M}\right)\right] .
$$

From the enthalpy field the liquid fraction $f$ can be obtained.

\section{Numerical discretisation}

The governing equations are discretised on a Cartesian mesh. The grids are non-orthogonal (the line joining the centroids of the neighbouring control volumes are non-orthogonal to their common face) in the mushy CVs and in the CVs which are close to the solid-liquid interface. The calculation of the diffusive flux in these $\mathrm{CV}$ s requires treatment which is similar to that in a complex domain. The details of discretisation of diffusive fluxes in a complex domain are discussed in Mathur \& Murthy (1997). Consider the calculation of the diffusive flux for the face ' $e$ ' of the CV in figure 4. This involves the calculation of the derivative $\phi_{x}$ on the face of the control volume. 


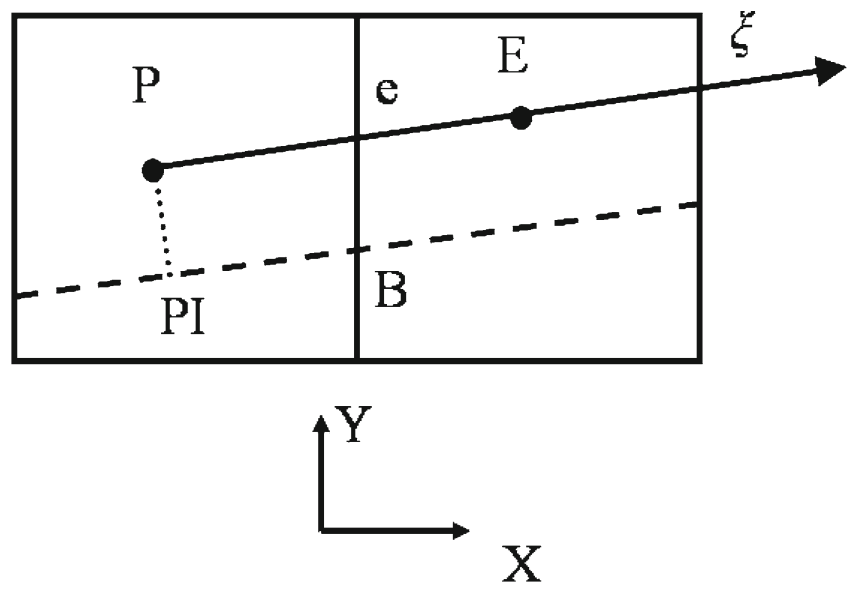

Figure 4. Sample control volume for discretisation.

Let $\xi$ denote a coordinate along the line joining the centroids of $\mathrm{CV}$, viz., ' $P$ ' and ' $E$ '. $\phi_{x}$ can be evaluated as

$$
\phi_{x}=\frac{\phi_{\xi}-\phi_{y} y_{\xi}}{x_{\xi}}
$$

The finite difference for various terms in the above equation can be written as

$$
x_{\xi}=\frac{x_{E}-x_{P}}{\Delta \xi}, \quad y_{\xi}=\frac{y_{E}-y_{P}}{\Delta \xi}, \quad \phi_{\xi}=\frac{\phi_{E}-\phi_{P}}{\Delta \xi}, \quad \phi_{y}=\frac{\phi_{\mathrm{A}}-\phi_{\mathrm{B}}}{y_{\mathrm{A}}-y_{\mathrm{B}}},
$$

where $\Delta \xi=\sqrt{\left(x_{E}-x_{P}\right)^{2}+\left(y_{E}-y_{P}\right)^{2}}$.

It can be seen from the equation (14) that calculation of $\phi_{y}$ involves the values of $\phi$ at points $\mathrm{A}$ and $\mathrm{B}$. The value of $\phi_{\mathrm{B}}$ can be calculated from the boundary condition at the solid-liquid interface. The value of $\phi_{\mathrm{A}}$ is obtained by inverse distance weighted interpolation from the neighbouring control volumes. For the mushy $\mathrm{CV}$, the existence of a solid-liquid interface demands an additional diffusive flux calculation at the interface. This diffusive flux requires the evaluation of the term $\phi_{n}$ where $n$ is a coordinate normal to the interface. This is obtained by dropping a perpendicular from the centroid ' $\mathrm{P}$ ' to the interface and evaluating $\phi_{n}$ as,

$$
\phi_{n}=\frac{\phi_{P I}-\phi_{P}}{\sqrt{\left(x_{P}-x_{P I}\right)^{2}+\left(y_{P}-y_{P I}\right)^{2}}} .
$$

The total diffusive transport for a given control volume can thus be written in a standard form as

$$
D=\sum_{E, W, N, S} d_{n b} \phi_{n b}+d_{I} \phi_{I}+S_{S}-d_{P} \phi_{P}
$$

where $S_{S}$ denotes the source term due to secondary flux and $d_{I} \phi_{I}$ denotes the contribution from the solid-liquid interface. It is possible that some of the $d_{n b}$ terms may be zero, for e.g., for the 
control volume in figure $4, d_{S}$ is zero. The convective terms are evaluated by first order upwind method. The final discrete form of the equation (11) can be written as

$$
\frac{\left(f\left(\phi_{P}-\phi_{I}\right)\right)-\left(f\left(\phi_{P}-\phi_{I}\right)\right)^{O}}{\Delta t} \Delta V=\sum_{E, W, N, S} a_{n b} \phi_{n b}+a_{I} \phi_{I}+S_{S}+S-a_{P} \phi_{P} .
$$

In the above equation, $S_{S}$ and $S$ denote the source term due to secondary flux and other body source terms, respectively.

\subsection{Solution of the Navier-Stokes equation}

The Navier-Stokes equations are solved on a collocated Cartesian grid. The solution of these equations requires the calculation of pressure force in addition to the solution of the convection diffusion equation. The final form of the momentum equation after discretisation of the convective and diffusive terms read as

$$
\begin{aligned}
& a_{P} u_{P}=\sum_{E, W, N, S} a_{n b} u_{n b}+S+S_{S}+F_{P X} \\
& a_{P} v_{P}=\sum_{E, W, N, S} a_{n b} v_{n b}+S+S_{S}+F_{P Y},
\end{aligned}
$$

where $F_{P X}$ and $F_{P Y}$ denotes the pressure force terms in the $x$ and the $y$ directions, respectively. To calculate the pressure force the pressures at the face of the control volumes are used. The pressure on a face such as face ' $e$ ' in figure 4 is obtained by linear interpolation of the pressures from centroids ' $P$ ' and ' $E$ '. The pressure at the solid-liquid interface is obtained by applying the Navier-Stokes equation at the interface which results in

$$
\frac{\partial P}{\partial n}=v \frac{\partial^{2} u_{n}}{\partial n^{2}}
$$

where $n$ is a coordinate normal to the interface. By finite difference,

$$
P_{P I}=P_{P}+v \Delta n \frac{\partial^{2} u_{n}}{\partial n^{2}}
$$

The pressure at the interface can be obtained. The pressure force in the $x$ and $y$ directions are

$$
\begin{gathered}
F_{P X}=P_{w} A_{w}-P_{e} A_{e}-\left(A_{e}-A_{w}\right) P_{P I} . \\
F_{P Y}=P_{s} A_{s}-P_{n} A_{n}-\left(A_{s}-A_{n}\right) P_{P I} .
\end{gathered}
$$

The areas $\left(A_{e}-A_{w}\right)$ and $\left(A_{s}-A_{n}\right)$ are the projected areas of the interface in the positive $x$ and $y$ directions, respectively.

\subsection{Momentum interpolation}

The governing equations are solved on a collocated grid. Interpolating the velocities at the centroids to obtain the velocities at the faces result in pressure checkerboarding. To overcome this difficulty, the momentum interpolation of Rhie \& Chow (1983) has been used. 
The momentum equations can be written as

$$
\begin{aligned}
& u=\tilde{u}+\frac{1}{a_{p}} F_{P X} . \\
& v=\tilde{v}+\frac{1}{a_{p}} F_{P Y} .
\end{aligned}
$$

In order to obtain the velocity at the face ' $e$ ' of the control volume ' $P$ ' in figure 4 momentum interpolation is followed. The face velocity can be written as

$$
u_{f e}=\tilde{u}_{f e}+\frac{1}{a_{p f e}} F_{P X},
$$

where

$$
F_{P X}=-\int \frac{\partial P}{\partial x} d V \approx-\frac{\partial P}{\partial x} \Delta V_{f e}
$$

Thus,

$$
u_{f e}=\tilde{u}_{f e}-\frac{\partial P}{\partial x} \frac{\Delta V_{f e}}{a_{p f e}} .
$$

The value of $\tilde{u}_{f e}, a_{p f e}, \Delta V_{f e}$ are obtained by linear interpolation of the corresponding values from ' $P$ ' and ' $E$ ' control volumes (figure 4). The derivative term $\frac{\partial P}{\partial x}$ is evaluated in a manner similar to that involved in the calculation of the diffusive term described in section 5 . The discrete form of the above equation is

$$
u_{f e}=\tilde{u}_{f e}-\frac{P_{E}-P_{P}}{\Delta \xi} \frac{\Delta V_{f e}}{x_{\xi} a_{p f e}}+S_{S P},
$$

where $S_{S P}$ contains the secondary pressure gradient term arising due to the non-orthogonality of the grid. The pressure at the centroids of the control volumes are obtained by the continuity equation by using SIMPLE method of Patankar (1980). The face velocity correction term can be written as,

$$
u_{f e}^{\prime}=-\frac{P_{E}^{\prime}-P_{P}^{\prime}}{\Delta \xi} \frac{\Delta V_{f e}}{x_{\xi} a_{p f e}},
$$

where $P^{\prime}$ is the pressure correction term. By substituting the face velocities in the continuity equation and incorporating the correction terms, an equation for the pressure correction is obtained. This pressure correction equation is solved and the pressures and velocities are corrected. The enthalpy equation is solved on the whole control volume. To calculate the diffusive and convective fluxes, temperatures at the centroids are used. This prevents any interpolation of temperature across the solid-liquid interface.

\subsection{Summary of the numerical procedure}

Assuming the velocity, temperature and the liquid fraction are available at a previous time, the following steps are followed

(a) Solve the momentum equations (equations (19) and (20)) and obtain $\tilde{u}$ and $\tilde{v}$.

(b) Obtain the face velocities by momentum interpolation using equation (30). 
(c) Solve the pressure correction equation and hence correct the face and the centroidal velocities (equation (31)).

(d) Solve the energy equations to obtain the temperatures in the liquid and the solid domain using equation (18).

(e) Solve the enthalpy equation to obtain the enthalpy field and hence obtain liquid fraction $f$ from equation (13).

(f) Using the newly obtained liquid fraction reconstruct the interface.

(g) Solve the above steps until convergence.

The form taken by the system of governing equations for the special case of one-dimension is mentioned in appendix B. Appendix B also contrasts the significant difference between the present method and the explicit enthalpy method of Tacke (1985) a popular method which also eliminates waviness.

\section{Results and discussions}

To benchmark the cell splitting method and to demonstrate that it can eliminate waviness produced by the enthalpy method, a simple 1-D problem is chosen. The problem consists of a semi-infinite slab $(0 \leq x<\infty)$, which is initially solid at its melting temperature. The left end is raised to a temperature $T_{W}$ which is greater than the melting point of the material $T_{M}$. The solid-liquid interface moves monotonously to the right.

The governing equations for this situation is given by

$$
\frac{\partial T}{\partial t}=\alpha \frac{\partial^{2} T}{\partial x^{2}} \quad \text { for } 0<x<s(t)
$$

where $s(t)$ denotes the position of the solid-liquid interface at time $t$. At the solid-liquid interface the boundary conditions are

and,

$$
T=T_{M}
$$

$$
-k \frac{\partial T}{\partial x}=\rho \Delta H_{M} \frac{d s(t)}{d t} .
$$

By introducing the following non-dimensional variables,

$$
X^{*}=\frac{x}{L_{S}}, \quad S=\frac{s}{L_{S}}, \quad \tau=\frac{\alpha t}{L_{S}^{2}}, \quad \theta=\frac{\left(T-T_{M}\right)}{\left(T_{W}-T_{M}\right)} .
$$

The governing equation becomes

$$
\frac{\partial \theta}{\partial \tau}=\frac{\partial^{2} \theta}{\partial X^{* 2}} .
$$

Along with

$$
\begin{gathered}
\theta=0 \\
-\frac{\partial \theta}{\partial X}=\frac{1}{\mathrm{St}} \frac{d S(t)}{d \tau} .
\end{gathered}
$$


At the solid-liquid interface here Stefan number St is defined by

$$
\mathrm{St}=\frac{C_{P}\left(T_{W}-T_{M}\right)}{\Delta H_{M}} .
$$

The analytical solution for the above problem was given by Neumann and has been reproduced in Crank (1984). The solution in the non-dimensional form is

$$
\theta=1-\frac{\operatorname{erf}\left(\frac{X}{2 \sqrt{\tau}}\right)}{\operatorname{erf}(\lambda)} \quad 0<X^{*}<S(\tau) .
$$

The position of the interface is given by

$$
S(\tau)=2 \lambda \sqrt{\tau}
$$

where $\lambda$ is obtained by solving the equation

$$
\frac{\mathrm{St}}{\exp \left(\lambda^{2}\right) \operatorname{erf}(\lambda)}=\lambda \sqrt{\pi} .
$$

A one-dimensional finite Cartesian computational domain of length equal to the length scale $L_{S}$ was chosen and was discretised into 10 equal spaced control volumes. The right end was imposed to be at the melting temperature. The temperatures of the points at non-dimensional distances of $X^{*}=0.25, X^{*}=0.5$ and $X^{*}=0.75$ are plotted as a function of time in figures 5-7. These figures show a comparison of the results obtained from the classical enthalpy method and the present cell-splitting method with the analytical solutions. It can be seen that the cell splitting method has eliminated the waviness.

Few comments can be made on the waviness of the enthalpy method. In the enthalpy method, the centroid of the control volume which is undergoing a phase change is anchored at the melting point. In this duration, the solid and the liquid domains experience constant temperature boundary conditions and tend to move towards a steady state. This explains the flattening of the temperature and thus the waviness. The waviness will be more pronounced if the interface takes more time to cross a control volume. For a given Stefan number the interface velocity decreases with time. Thus, the waviness for a given Stefan number is more at the location $X^{*}=0.75$ as compared to the location at $X^{*}=0.25$. This can be seen from figures 5-7. Similarly, at a fixed location, the velocity with which a solid-liquid interface moves through this location increases with the Stefan number. This explains the decrease in the waviness with increase in the Stefan number.

It can be seen from figures 5-7 that the classical enthalpy method seems to constantly under predict the temperature for higher Stefan numbers. This can be explained as follows. Let $\theta_{a}$, $\theta_{b}$ denote the temperature of a control volume $\mathrm{k}$ predicted by the classical enthalpy method at the start $\left(\tau_{a}\right)$ and the end $\left(\tau_{b}\right)$ of a wavy cycle, respectively (figure 5a). This wavy cycle will correspond to the melting of a control volume $\mathrm{i}+1$ (figure 5b). $\theta_{a}$ and $\theta_{b}$ will be roughly equal to the steady state temperatures reached by the control volume $\mathrm{k}$ when centroids of the control volumes $\mathrm{i}$ and $\mathrm{i}+1$ are set at the melting temperature. For a duration $\tau_{a}<\tau<\tau^{*}$, where $\tau *$ is the time at which the interface is at the centroid of the control volume at $i+1$, the analytical results at control volume $\mathrm{k}$ will be lower than $\theta_{b}$ and vice versa for $\tau *<\tau<\tau_{b}$. Further, if $\tau * *$ 


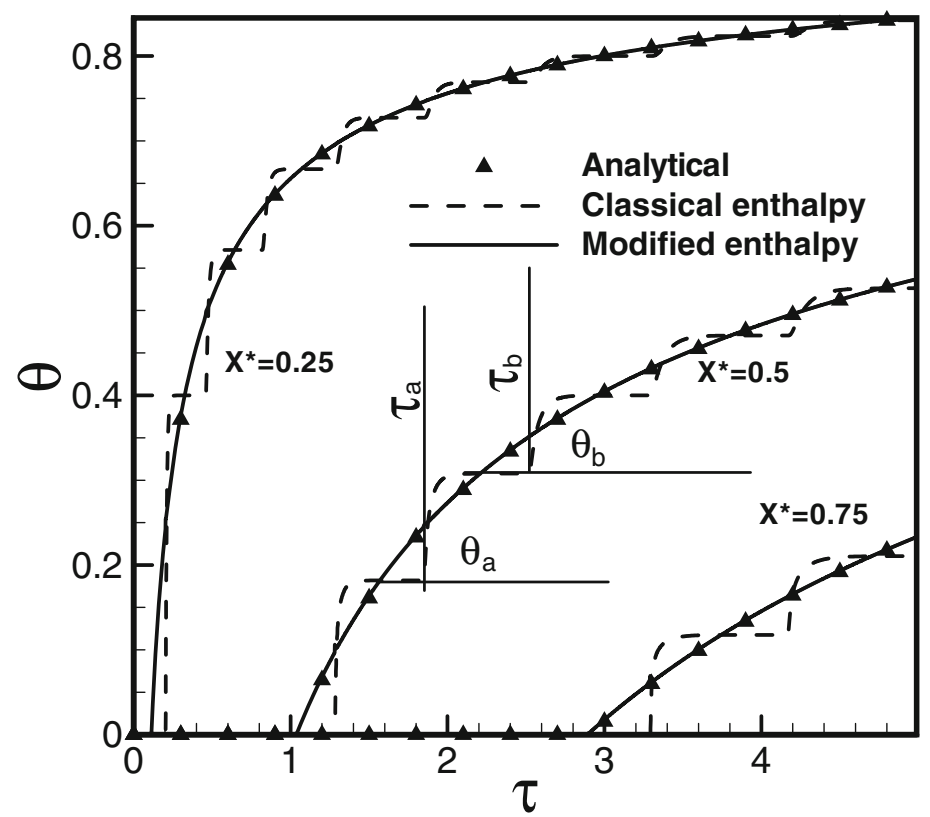

(a)
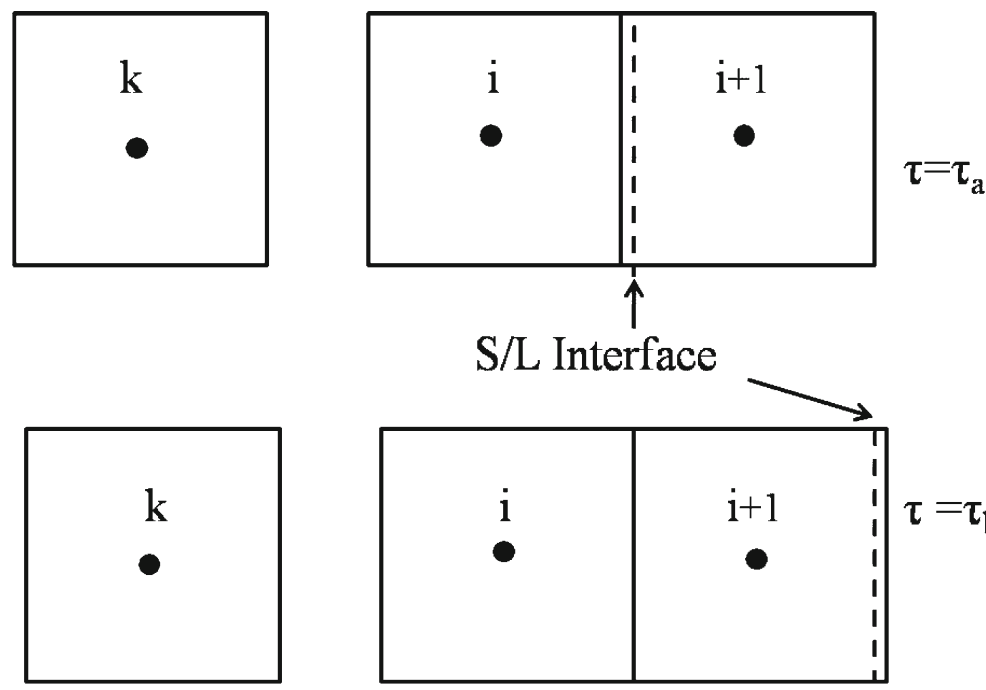

\section{S/L Interface}

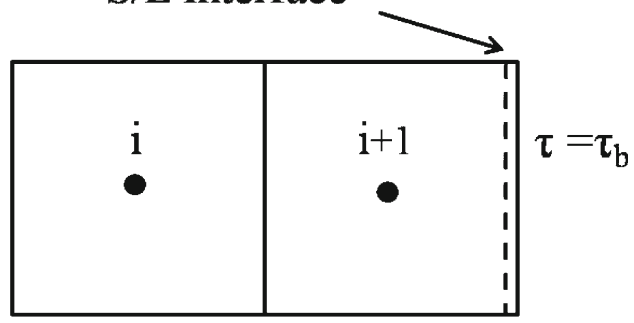

(b)

Figure 5. (a) Comparison of the temperature at the mid point of the domain as a function of time for Stefan number of 0.1. (b) The control volumes with interface for the explanation of waviness.

denotes the time taken for the control volume $\mathrm{k}$ to reach the pseudo steady state corresponding to melting temperature being imposed at the centroid of the control volume $\mathrm{i}+1$, for $\tau^{* *}<\tau<\tau_{b}$ the temperature of the control volume will be $\theta_{b}$. If $\left(\tau^{* *}-\tau_{a}\right)$ is less than $\left(\tau^{*}-\tau_{a}\right)$, for some duration the temperature obtained from the enthalpy method will be over predicted. If however 


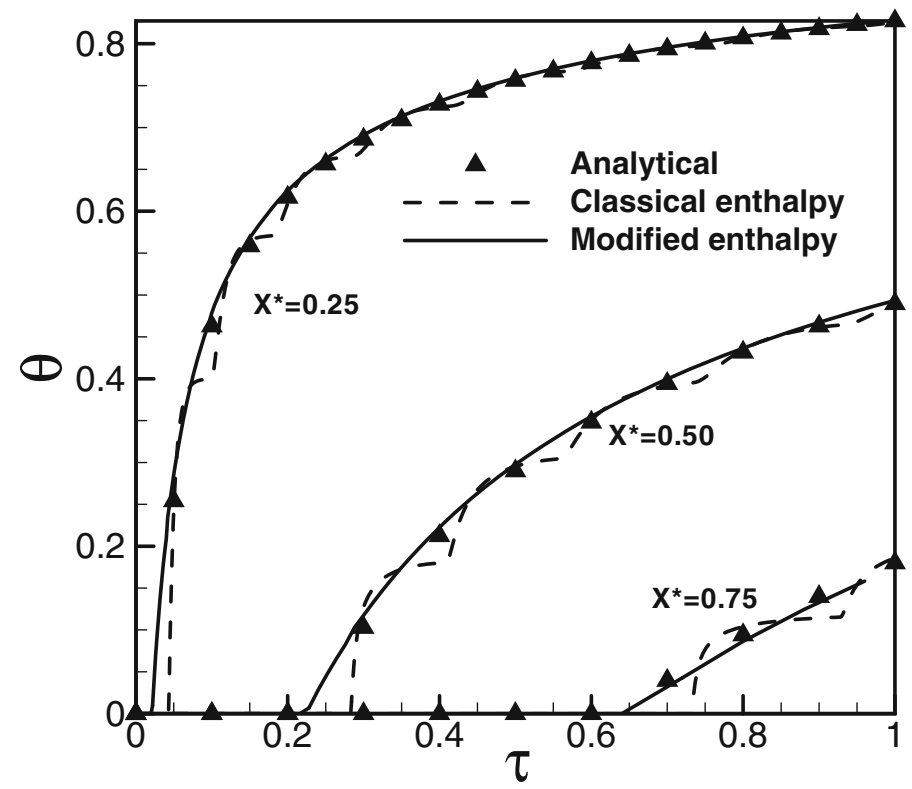

Figure 6. Comparison of the temperature at the mid point of the domain as a function of time for Stefan number of 0.5 .

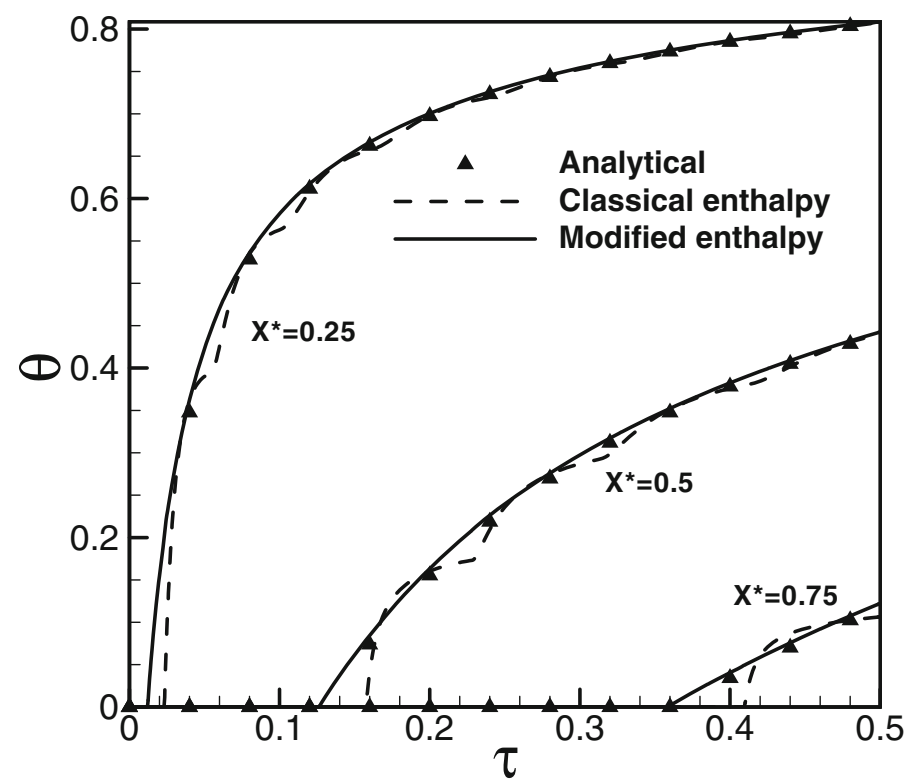

Figure 7. Comparison of the temperature at the mid point of the domain as a function of time for Stefan number of 1.0 . 
$\left(\tau * *-\tau_{a}\right)$ is sufficiently greater than $\left(\tau^{*}-\tau_{a}\right)$ the temperature obtained from the enthalpy method will under predict for the entire duration $\theta_{a}<\theta<\theta_{b}$.

For very low Stefan numbers (such as $\mathrm{St}=0.1$ ), the velocity for the solid-liquid interface is lower. This implies that the time taken to reach steady state in a control volume $\mathrm{k}$ is significantly lower than the time taken for the control volume $\mathrm{i}+1$ to undergo phase change, i.e., $\left(\tau * *-\tau_{a}\right)<<\left(\tau_{b}-\tau_{a}\right)$. Thus, for some duration, the enthalpy method over-predicts the temperature (figure 5). However, for larger Stefan numbers (such as $\mathrm{St}=1$ ) the time taken to reach steady state is larger than the time taken for the control volume to undergo phase change. This may be inferred from figure 7 where the curvilinear profile does not reach an asymptotic straight line as seen in figure 5. This explains the reason behind the under prediction of the temperature by the enthalpy method in comparison to the analytical solution for the position $X^{*}=0.25$ in figure 7 .

It may be noted that in 2 or 3 dimensions when the interface is not oriented along a coordinate axis the waviness in the enthalpy method will be considerably reduced. In such cases, the control volumes which are undergoing phase change melt at different rates thereby avoiding synchronisation of the imposition of the artificial boundary condition on the centroids of all the control volumes undergoing phase change. This will reduce waviness. To demonstrate the reduction in the waviness of the classical enthalpy method and to further benchmark the cell splitting method, 2-d conduction melting problems are simulated. Since no true 2-d analytical solutions are available, two pseudo 2-d problems were created from 1-d exact solutions. The first problem consists of a 2-dimensional domain embedded in the semi-infinite slab melting problem (considered previously) as shown in figure 8. The Cartesian domain has a non-dimensional width and length of 1 . The temperatures on the boundary of the Cartesian domain are calculated from the analytical solution and are imposed as the boundary conditions. The solid-liquid interface in such a situation will be oriented at an angle of $\theta$ with respect to the $x$ axis of the 2-d domain. The domain is discretised into $10 \times 10$ control volumes. The temperature of the centre of the $2-\mathrm{d}$ domain is plotted as a function of time in figure 9 for 2 different orientations of the solid-liquid interface.

To benchmark the present method when the solid-liquid interface is curved, melting of an infinite cylinder due to a line heat source is considered (figure 10). The problem consists of an

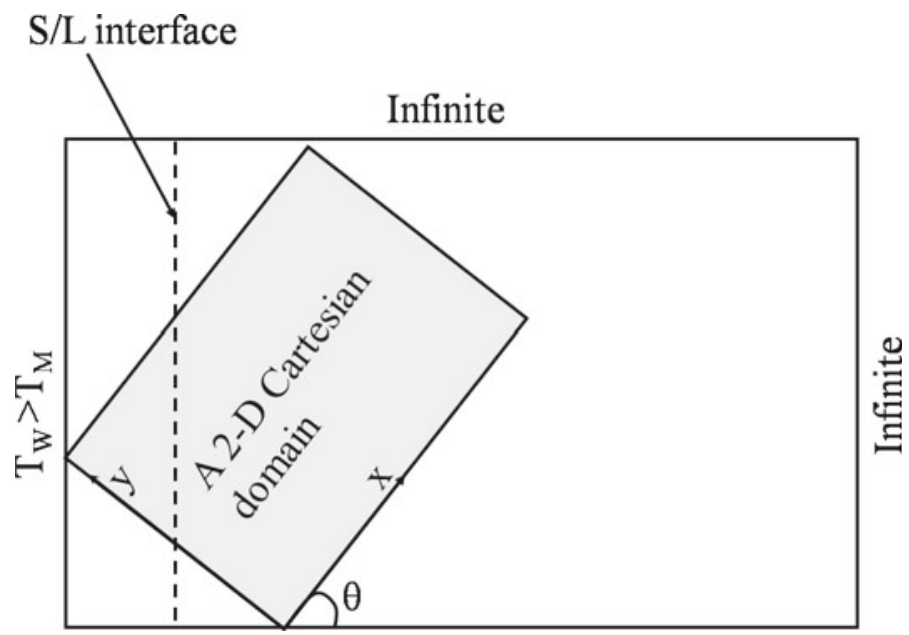

Figure 8. The 2-d domain chosen for melting with straight interface. 


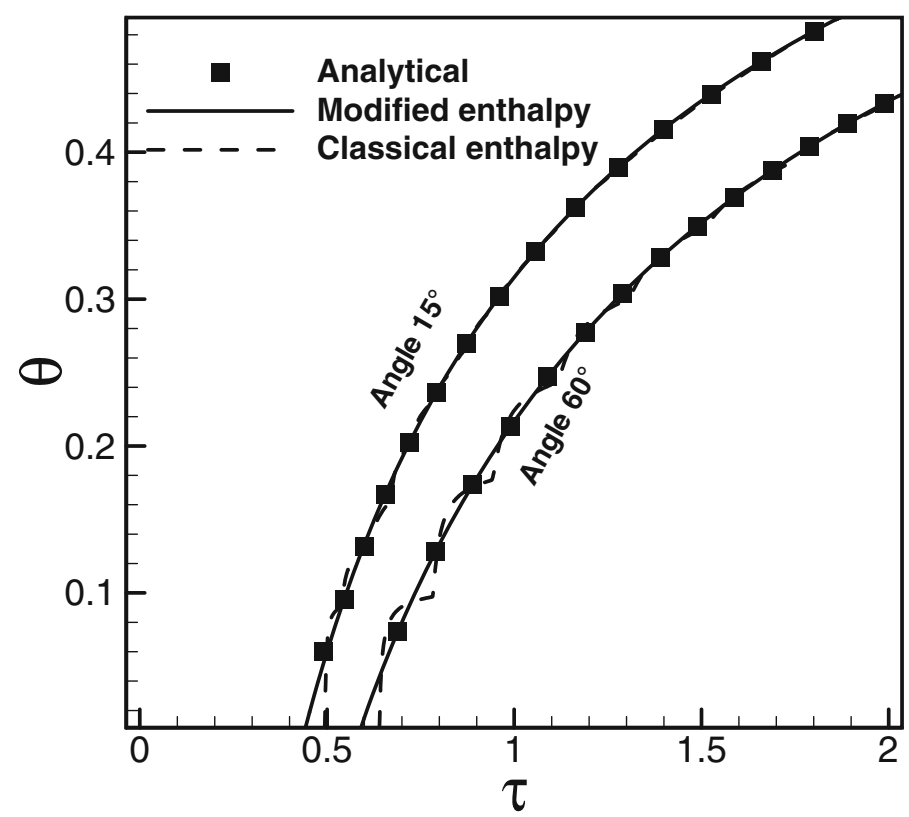

Figure 9. Temperature time plot at the centre of a two-dimensional domain for various angle of orientation of the interface. Stefan number $=1.0$.

infinite cylinder initially solid at its melting point. A line heat source aligned along the $z$ direction is switched on at time $t>0$. The solid-liquid interface which will be an infinite cylinder with axis along the $z$ direction will expand as time progresses. The governing equation for the situation will be

$$
\frac{\partial T}{\partial t}=\alpha \frac{1}{r} \frac{\partial}{\partial r}\left(r \frac{\partial T}{\partial r}\right) \quad 0<r<R(t)
$$

where $R$ is the location of the solid-liquid interface. At the origin

$$
\operatorname{Lim}_{r \rightarrow 0}\left(-2 \pi r k \frac{\partial T}{\partial r}\right)=Q
$$

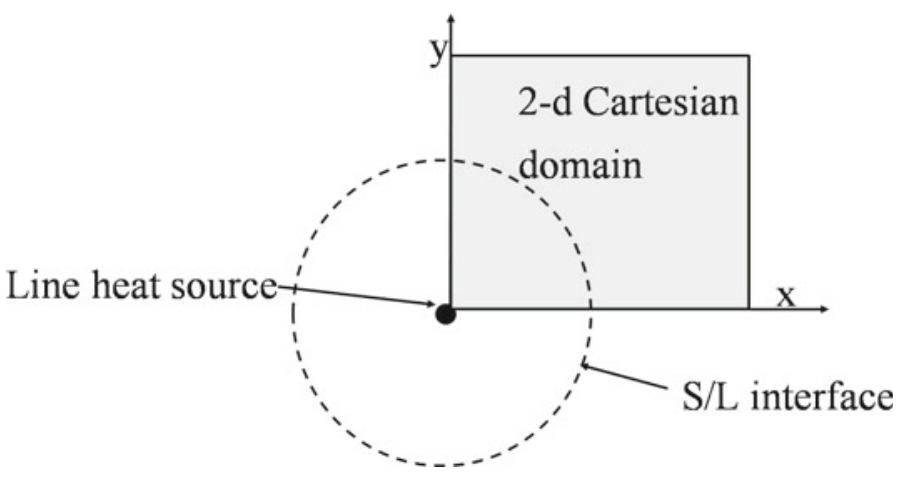

Figure 10. The 2-d domain chosen for melting with circular interface. 
where $Q$ is the strength of the line heat source. At the solid-liquid interface

$$
\begin{gathered}
T=T_{M}, \\
-k \frac{\partial T}{\partial r}=\rho \Delta H_{M} \frac{d R(t)}{d t} .
\end{gathered}
$$

By introducing the following variables,

$$
r^{*}=\frac{r}{L_{S}}, \quad R^{*}=\frac{R}{L_{S}}, \quad \tau=\frac{\alpha t}{L_{S}^{2}}, \quad \theta=\frac{\left(T-T_{M}\right)}{\Delta T_{r e f}}, \quad \Delta T_{r e f}=\frac{Q}{k}
$$

The governing equation in non-dimensional form is

$$
\frac{\partial \theta}{\partial \tau}=\frac{1}{r^{*}} \frac{\partial}{\partial r^{*}}\left(r^{*} \frac{\partial \theta}{\partial r^{*}}\right) \quad 0<r^{*}<R^{*}(\tau) .
$$

At the origin

$$
\operatorname{Lim}_{r^{*} \rightarrow 0}\left(-2 \pi r^{*} \frac{\partial \theta}{\partial r^{*}}\right)=1
$$

At the solid-liquid interface

$$
\begin{gathered}
\theta=0, \\
-\frac{\partial \theta}{\partial r^{*}}=\frac{1}{\mathrm{St}} \frac{d R^{*}(\tau)}{d \tau},
\end{gathered}
$$

where

$$
\mathrm{St}=\frac{C_{P} \Delta T_{r e f}}{\Delta H_{M}} .
$$

The analytical solution for this situation was given by Paterson (1952) which in non-dimensional form is

$$
\theta=\frac{1}{4 \pi}\left(E i\left(-\lambda^{2}\right)-E i\left(-\frac{r^{* 2}}{4 \tau}\right)\right) \quad 0<r^{*}<R(\tau) .
$$

The position of the interface is given by

$$
R^{*}(\tau)=2 \lambda \tau^{\frac{1}{2}}
$$

where $\lambda$ is obtained by solving the equation

$$
-\frac{1}{4 \pi} e^{-\lambda^{2}}=\frac{\lambda^{2}}{\mathrm{St}}
$$

In the above equations the $E i$ is the exponential integral defined as

$$
E i(x)=\int_{-\infty}^{x} \frac{e^{t}}{t} d t
$$

A 2-d Cartesian domain which has a unit non-dimensional width and length is carved out as shown in figure 10. For this domain, the temperature at the boundaries of the domain is calculated from the analytical solutions and is imposed as boundary conditions. 
The domain is discretised into $10 \times 10$ control volumes. The temperature of the centre of the 2-d domain is plotted as a function of time in figure 11. It can be seen that the temperature predicted by the cell-splitting method shows no waviness and matches well with the analytical solutions. It can also be seen that the waviness in the enthalpy method has been reduced.

To demonstrate the applicability of the method to the simulation of melting with natural convection, two bench mark problems were chosen, the first involved melting of gallium a low Prandtl substance $(\operatorname{Pr}=0.021)$ and second involved melting of $n$-octadecane a high Prandtl substance $(\mathrm{Pr}=57)$. Both these simulation involved melting in a rectangular container, where the substance was initially solid at its melting point. At time $t=0$ the left wall was raised to a temperature $T_{W}>T_{M}$. The top and the bottom walls are insulated. The density difference between the hot and the cold fluids causes convection currents in the fluid. These convective currents alter the temperature distribution by creating higher temperatures near the top of the cavity. This increases the melting rate at the top and causes a distortion in the shape of the solidliquid interface. In order to model the effect of density difference, Boussinesq approximation was used. Figure 12a shows the solid-liquid interface at various times obtained from the present method and the enthalpy porosity method of Brent et al (1988) with a mushy zone constant of $10^{5}$ and $10^{10}$ for the melting of a gallium slab. It can be seen that although the deviation between the solid-liquid interface obtained from enthalpy-porosity method and the present cell-splitting method are small, the solid-liquid interface predicted by the present method seems to be sandwiched between the enthalpy-porosity method for mushy zone constants of $10^{5}$ and $10^{10}$ (in later times). Both the simulations were performed on a $80 \times 80$ grid. Thus, by tuning these mushy zone constants better agreements between the present method and the enthalpy-porosity method can be obtained. It may be emphasized that although the predictions of the enthalpy-porosity method seems less sensitive to the mushy zone constants in this situation, significant sensitivity to mushy zone constants has been reported in the melting of a vertical cylinder by Shmueli

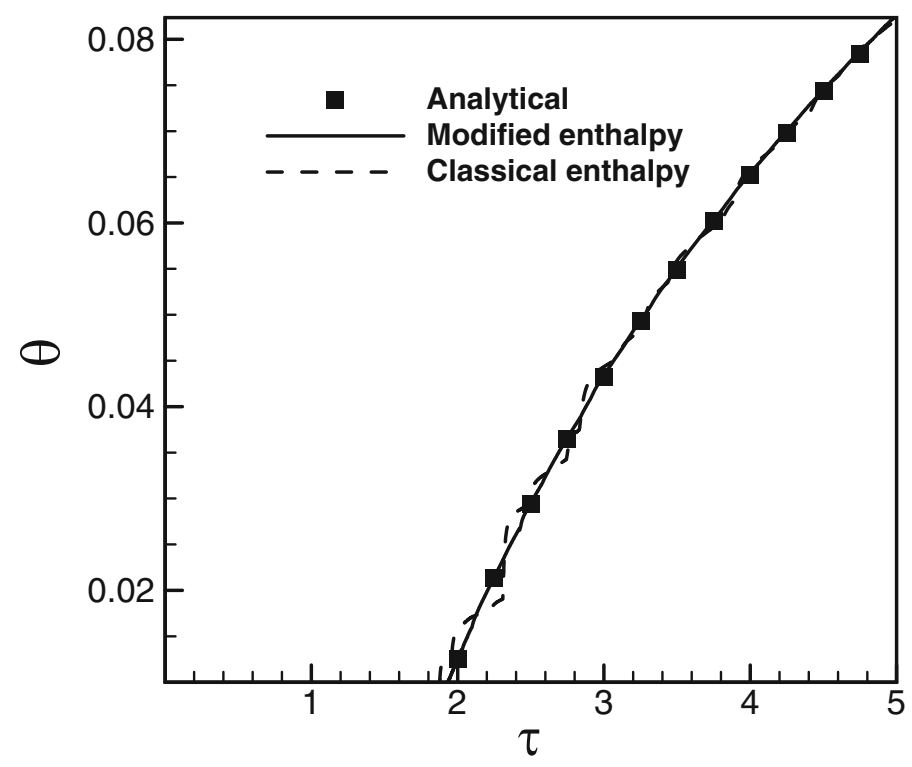

Figure 11. Temperature time plot at the centre of the two-dimensional domain for Stefan number $=1.0$. 


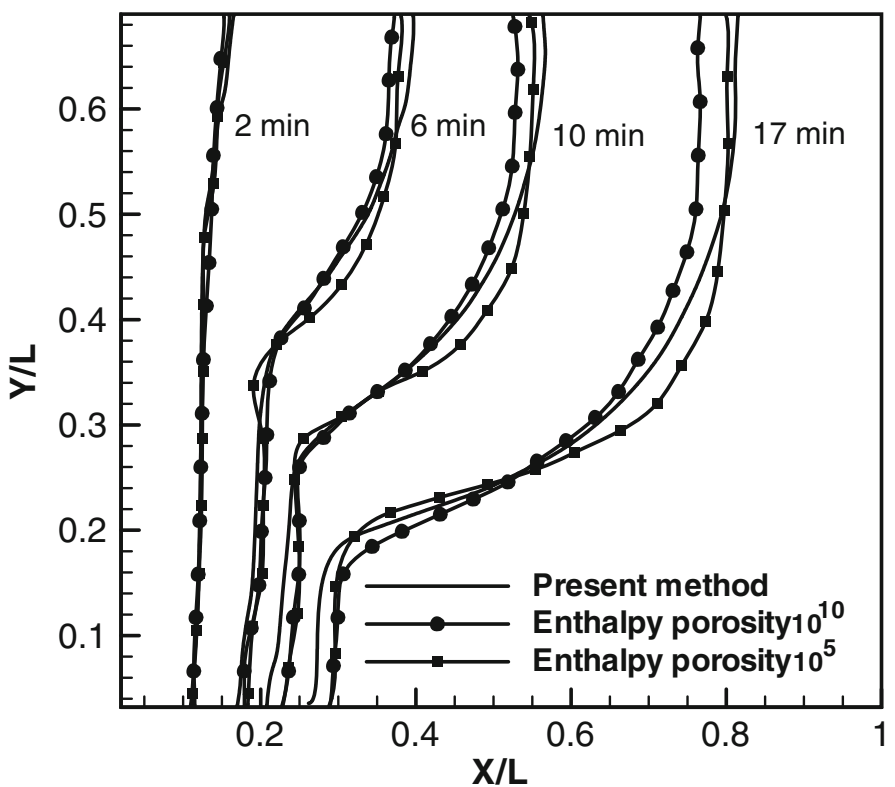

(a)

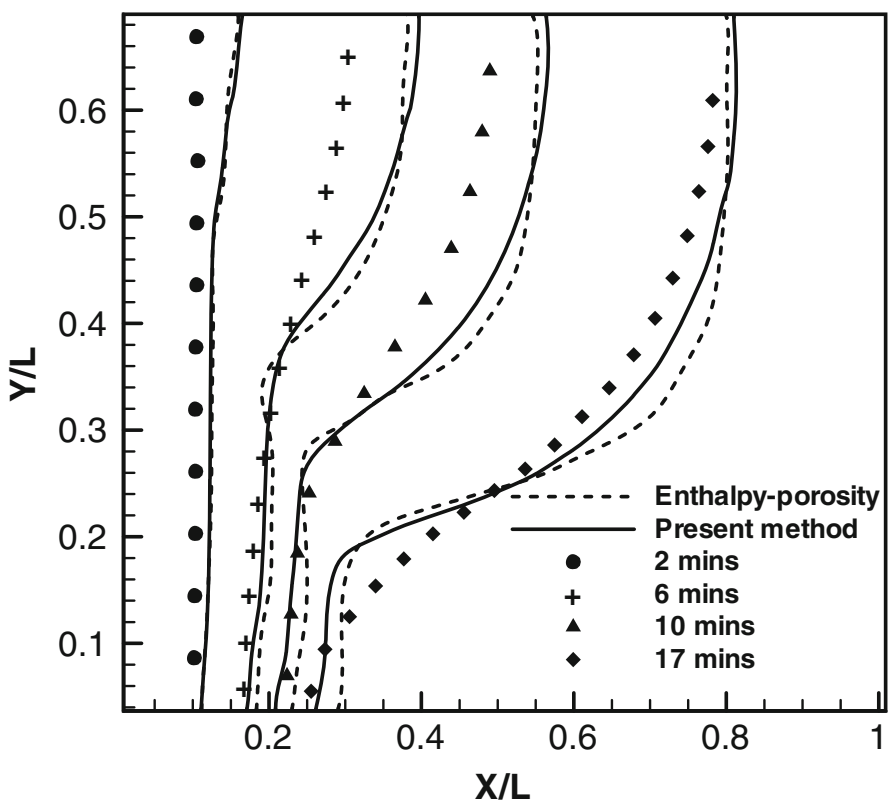

(b)

Figure 12. Comparison of the numerical prediction with the (a) enthalpy-porosity model of Brent et al (1988) for mushy zone constants of $10^{5}$ and $10^{10}$. (b) Enthalpy-porosity model of Brent et al (1988) for mushy zone constants of $10^{5}$ and experimental results of Gua \& Viskanta (1986) for an aspect ratio of 0.714 . 


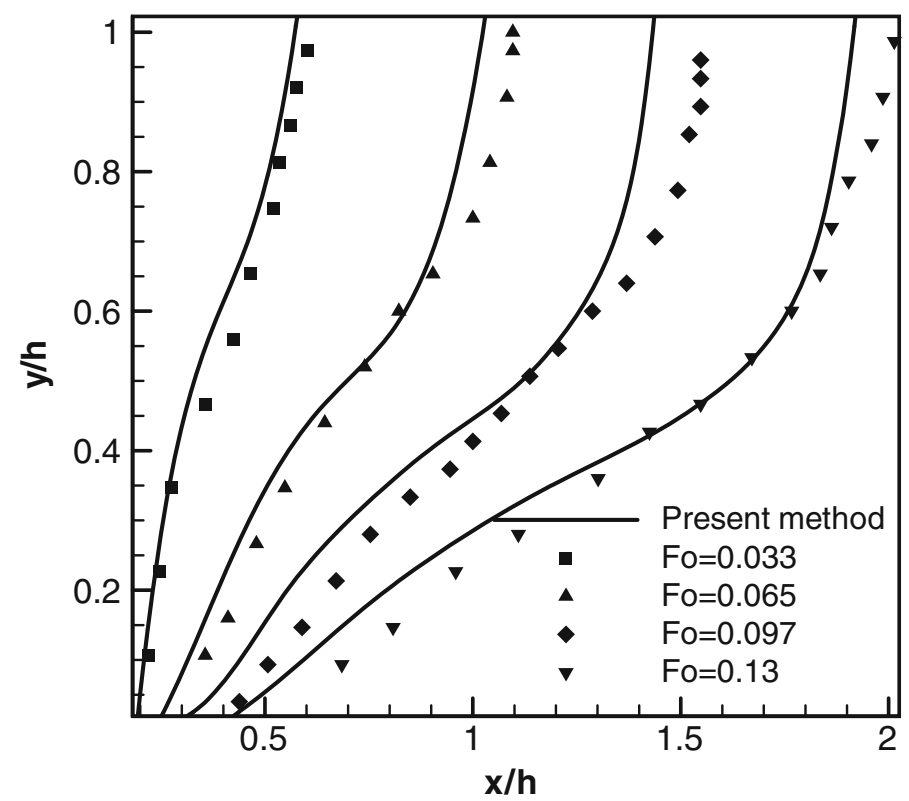

Figure 13. Comparison of the numerical prediction with the experimental results of Okada (1984). $\mathrm{Ra}=1.39 \times 10^{6}, \mathrm{St}=0.192$.

et al (2010). The present method eliminates the need for these mushy zone constant. Figure $12 \mathrm{~b}$ shows the predictions of the solid-liquid interface present method with the experimental results of Gua \& Viskanta (1986). It can be seen that the results agree reasonably well. Figure 13 shows the solid-liquid interface for the melting of $n$-octadecane. The numerical results carried out on a $80 \times 80$ grid compare well with experimental results of Okada (1984).

\section{Conclusions}

In this study, a cell splitting method based on enthalpy method for simulation of melting and solidification is presented. The cell splitting method is demonstrated to eliminate waviness present in the classical enthalpy method. The method also eliminates the need for any mushy zone constants for simulating melting with natural convection. Thus for pure materials, the method has eliminated the need for empirical constants. The method has been successfully benchmarked against both one and two-dimensional melting problems with and without natural convection.

\section{Appendix A}

In this section, we prove that the interface constructed is second order accurate. Let $\Gamma(x, y)=0$ denote the equation of the actual interface. Consider the situation shown in figure 14 . We assume that in the neighbourhood of $x=0$ the curve $\Gamma(x, y)$ can be written as, $y=f(x)$. Let $y=$ $g(x)=m x+c$ denote the straight line approximation to this curve. 


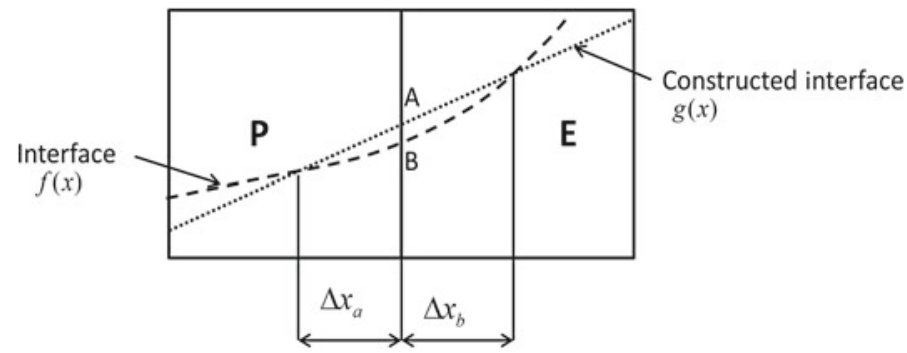

Figure 14. The error analysis for interface construction method.

The error $e$ will be defined as the distance between the points A and B in figure 14. The error is thus

$$
e=|g(0)-f(0)|=|c-f(0)| .
$$

The approximate interface $g(x)$ will be chosen by the present interface construction method such that ratio in which it divides the control volumes ' $P$ ' and ' $E$ ' are same as that by $f(x)$.

The above mentioned conditions will hold true only if $f(x)$ and $g(x)$ intersect each other at least once in ' $P$ ' and ' $E$ '.

Let $x_{a}$ and $x_{b}$ denote these points of intersection such that $0<x_{b}<h$ and $-h<x_{a}<0$, where $h$ denotes the dimension of the control volume in the $x$ direction. By Taylor series

$$
f(x)=f(0)+\frac{f^{\prime}(0)}{1 !} x+\frac{f^{\prime \prime}(0)}{2 !} x^{2}+\ldots \ldots
$$

At the points $x_{a}$ and $x_{b}$

$$
\begin{aligned}
& f\left(x_{a}\right)=g\left(x_{a}\right) \\
& f\left(x_{b}\right)=g\left(x_{b}\right) .
\end{aligned}
$$

Thus,

$$
\begin{aligned}
& m x_{a}+c=f(0)+\frac{f^{\prime}(0)}{1 !} x_{a}+\frac{f^{\prime \prime}(0)}{2 !} x_{a}^{2}+\ldots . . \\
& m x_{b}+c=f(0)+\frac{f^{\prime}(0)}{1 !} x_{b}+\frac{f^{\prime \prime}(0)}{2 !} x_{b}^{2}+\ldots . .
\end{aligned}
$$

Multiplying equation (60) by $x_{b}$ and equation (61) by $x_{a}$ and subtracting,

$$
\left(x_{b}-x_{a}\right)(c-f(0))=\frac{f^{\prime \prime}(0)}{2 !} x_{a} x_{b}\left(x_{b}-x_{a}\right)+\ldots . .+\frac{f^{n}(0)}{n !} x_{a} x_{b}\left(x_{b}^{n}-x_{a}^{n}\right) .
$$

The above equation can be simplified into,

$$
c-f(0)=\frac{f^{\prime \prime}(0)}{2 !} x_{a} x_{b}+\ldots \ldots+\frac{f^{n}(0)}{n !} x_{a} x_{b}\left(\sum_{0}^{n-2} x_{a}^{r} x_{b}^{n-2-r}\right) .
$$

Taking the modulus of the function

$$
|c-f(0)| \leq\left|\frac{f^{\prime \prime}(0)}{2 !} x_{a} x_{b}\right|+\ldots \ldots+\left|\frac{f^{n}(0)}{n !} x_{a} x_{b}\left(\sum_{0}^{n-2} x_{a}^{r} x_{b}^{n-2-r}\right)\right|+\ldots \ldots
$$


Since

$$
\begin{gathered}
\left|x_{a}\right| \leq h \quad \text { and } \quad\left|x_{b}\right| \leq h, \\
|c-f(0)| \leq\left|\frac{f^{\prime \prime}(0)}{2 !}\right| h^{2}+\ldots \ldots+\left|\frac{f^{n}(0)}{(n-1) !}\right| h^{n}+\ldots \ldots .
\end{gathered}
$$

Thus from equations (57) and (66)

$$
e=O\left(h^{2}\right)
$$

\section{Appendix B}

In this section, the form taken by the present cell splitting method for one-dimension situation is presented. The significant differences between the present scheme and the explicit enthalpy scheme of Tacke (1985) which eliminate waviness are also highlighted. Consider a stencil of control volume as show in figure 15. The solid-liquid interface is assumed to be present in the control volume ' $P$ ' while control volume ' $E$ ' is liquid and ' $W$ ' is solid. The centroids of the liquid and the solid control volumes are denoted by $X^{L}$ and $X^{S}$, respectively. The liquid fractions at the east and the west face of the control volume are denoted by $f_{e}$ and $f_{w}$, respectively. For the sake of clarity, the temperatures in the solid and the liquid domain are distinguished by the superscripts ' $S$ ' and ' $L$ ', respectively.

The differential form of the energy equation for the liquid domain obtained by suitably substituting for $\phi$ in equation (11) is

$$
\cdot \frac{d}{d t} \int_{V} \rho C_{P}\left(T^{L}-T_{M}\right) d V=\int_{A} k \nabla T^{L} \cdot \hat{n} d A+\int_{A_{I}} k \nabla T^{L} \cdot \hat{n} d A .
$$

Equation (68) can be discretised for the control volume shown in figure 15 as

$$
a_{P}^{L} T_{P}^{L}=a_{E}^{L} T_{E}^{L}+a_{W}^{L} T_{W}^{L}+a_{I}^{L} T_{M}+b
$$

where

$$
\begin{array}{cc}
a_{E}^{L}=f_{e} \frac{k}{x_{E}^{L}-x_{P}^{L}}, & a_{W}^{L}=f_{w} \frac{k}{x_{P}^{L}-x_{W}^{L}}, \\
a_{I}=\left\{\begin{array}{cc}
\frac{k}{x_{I}-x_{P}^{L}} & 0<f_{p}<1 \\
0 & f_{P}=0, f_{P}=1
\end{array}\right.
\end{array}
$$

\begin{tabular}{|c|cc:c|c|}
\multicolumn{1}{c}{ Liquid } & \multicolumn{2}{c}{ Interface } & \multicolumn{2}{c|}{ Solid } \\
\hline $\mathrm{W}$ & & $\mathrm{P}$ & $\bullet$ & $\mathrm{E}$ \\
$\bullet$ & $\bullet$ & & $\bullet$ & $\bullet$ \\
$\mathrm{X}_{\mathrm{W}}^{\mathrm{L}}$ & $\mathrm{X}_{\mathrm{P}}^{\mathrm{L}}$ & $\mathrm{X}_{\mathrm{P}}^{\mathrm{S}}$ & & $\mathrm{X}_{\mathrm{E}}^{\mathrm{S}}$ \\
\hline
\end{tabular}

Figure 15. The stencil for the control volume discretisation. 


$$
\begin{gathered}
a_{P}^{L}=a_{E}^{L}+a_{W}^{L}+a_{I}^{L}+\frac{\rho C_{P} f_{P} \Delta x_{P}}{\Delta t} \\
b=\frac{\rho C_{P} f_{P} \Delta x_{P}}{\Delta t} T_{M}+\frac{\rho C_{P} f_{P}^{O} \Delta x_{P}}{\Delta t}\left(T^{O^{L}}-T_{M}\right) .
\end{gathered}
$$

In the above equations the superscript ' $O$ ' denotes the values in the previous time steps and $\Delta x_{P}$ denotes the width of the control volume ' $P$ ' in figure 15 . In the situation shown in figure 15 where the liquid is not present on the east face $a_{E}^{L}=0$. A similar equation can be derived to obtain the temperature in the solid domain.

The enthalpy form of the energy equation (12) is

$$
\frac{d}{d t} \int_{V} \rho h d V+\int_{A_{\mathrm{C}}} \rho h \mathbf{V} . \hat{n} d A=\int_{A_{\mathrm{C}}} k \nabla T . \hat{n} d A .
$$

which can be written in a discrete form of as

$$
\rho \frac{h_{P}-h_{P}^{O}}{\Delta t}=a_{E}^{L}\left(T_{E}^{L}-T_{P}^{L}\right)+a_{E}^{S}\left(T_{E}^{S}-T_{P}^{S}\right)+a_{W}^{L}\left(T_{W}^{L}-T_{P}^{L}\right)+a_{W}^{S}\left(T_{W}^{S}-T_{P}^{S}\right),
$$

where $a_{E}^{S}$ and $a_{W}^{S}$ the coefficients obtained by solving the energy equation in the solid domain. From the enthalpy equation the liquid fraction can be recovered from,

$$
h_{P}=C_{P} f_{P}\left(T_{P}^{L}-T_{M}\right)+C_{P}\left(1-f_{P}\right)\left(T_{P}^{S}-T_{P}\right)+f_{P} \Delta H_{M},
$$

which asserts that the enthalpy of a control volume is composed of its sensible and latent heat parts.

Tacke (1985) had proposed an explicit enthalpy scheme which eliminates waviness in the enthalpy method. The significant difference between the schemes of the present cell splitting method with that of Tacke (1985) are

(a) In the method by Tacke (1985) the diffusive flux on the west face of the control volume for e.g., was discretised as

$$
Q_{W}=-k \frac{T_{M}-T_{W}}{X_{I}-X_{W}}
$$

while in the present method the diffusive flux is discretised as

$$
Q_{W}=-k \frac{T_{P}^{L}-T_{W}}{X_{P}^{L}-X_{W}}
$$

(b) In the method by Tacke (1985) the temperature of the solid and liquid domains which are necessary to calculate the sensible heat in the equation (76) are obtained by linear interpolation of the temperatures $T_{M}, T_{E}$ and $T_{M}, T_{W}$. While in the present method these temperature are obtained by solving the energy equation (69). 


\section{Nomenclature}

\begin{tabular}{|c|c|}
\hline$A$ & Area of a face of a control volume, $\mathrm{m}^{2}$ \\
\hline$A_{I}$ & Area of the interface part of a control volume, $\mathrm{m}^{2}$ \\
\hline$A_{\mathrm{C}}$ & Area of the Cartesian part of a control volume, $\mathrm{m}^{2}$ \\
\hline$a$ & Coefficients appearing in a transport equation \\
\hline$b$ & Source term in discretised equation \\
\hline$C_{P}$ & Specific heat, $\mathrm{J} / \mathrm{kg}-\mathrm{K}$ \\
\hline$C$ & $y$ intercept of the constructed interface, $\mathrm{m}$ \\
\hline$D$ & Net diffusion into a control volume \\
\hline$d$ & Coefficients appearing in a diffusion equation \\
\hline$e$ & $\begin{array}{l}\text { Error between the actual and the predicted } \\
\text { location of the interface }\end{array}$ \\
\hline$f$ & Liquid fraction \\
\hline$f(x)$ & Equation of the interface \\
\hline$F_{P X} F_{P Y}$ & Force due to pressure in $x$ and $y$ direction, $\mathrm{N}$ \\
\hline$g$ & Acceleration due to gravity, $\mathrm{m} / \mathrm{s}^{2}$ \\
\hline$g(x)$ & Equation of the constructed interface \\
\hline$H$ & Enthalpy, J/kg \\
\hline$h$ & Width of the control volume in appendix $\mathrm{A}, \mathrm{m}$ \\
\hline$k$ & Thermal conductivity, $\mathrm{W} / \mathrm{m}-\mathrm{K}$ \\
\hline$L_{S}$ & A characteristic length scale, $\mathrm{m}$ \\
\hline$m$ & Slope of the constructed interface \\
\hline$N$ & Direction normal to the solid-liquid interface, $\mathrm{m}$ \\
\hline$\hat{n}$ & Unit vector normal to a face \\
\hline$P$ & Pressure, $\mathrm{N} / \mathrm{m}^{2}$ \\
\hline$P^{\prime}$ & Pressure correction, $\mathrm{N} / \mathrm{m}^{2}$ \\
\hline$Q$ & Strength of a line heat source, $\mathrm{W} / \mathrm{m}$ \\
\hline$q_{D}$ & Net diffusion into a control volume \\
\hline$r$ & Radial coordinate, $\mathrm{m}$ \\
\hline$r^{*}$ & Non-dimensional radial coordinate \\
\hline$R$ & Radial location of the solid-liquid interface, $\mathrm{m}$ \\
\hline$R^{*}$ & $\begin{array}{l}\text { Radial location of the solid-liquid interface } \\
\text { in non-dimensional form }\end{array}$ \\
\hline$S$ & Volumetric source terms in a transport equation \\
\hline$S(\tau)$ & $\begin{array}{l}\text { Non-dimensional position of the solid-liquid } \\
\text { interface along the } x \text { direction }\end{array}$ \\
\hline$S_{S}$ & $\begin{array}{l}\text { Source term due to secondary gradient in } \\
\text { transport equation }\end{array}$ \\
\hline$S_{S P}$ & $\begin{array}{l}\text { Source term due to secondary gradient in } \\
\text { pressure, } \mathrm{m} / \mathrm{s}\end{array}$ \\
\hline$s(t)$ & $\begin{array}{l}\text { Position of the solid-liquid interface along } \\
\text { the } x \text { direction, } \mathrm{m}\end{array}$ \\
\hline$T$ & Temperature, $\mathrm{K}$ \\
\hline$T_{r e f}$ & A reference temperature, $\mathrm{K}$ \\
\hline$T_{M}$ & Melting point temperature, $\mathrm{K}$ \\
\hline$T_{W}$ & Temperature at the wall, $\mathrm{K}$ \\
\hline$t$ & Time, $\mathrm{s}$ \\
\hline
\end{tabular}


$(u, v) \quad$ Velocity coordinates, $\mathrm{m} / \mathrm{s}$

$\tilde{u}, \tilde{v} \quad$ Tilde velocity used in momentum interpolation, $\mathrm{m} / \mathrm{s}$

$V \quad$ Volume of a control volume, $\mathrm{m}^{3}$

V Velocity vector, $\mathrm{m} / \mathrm{s}$

$\mathbf{V}_{\mathbf{g}} \quad$ Velocity of the grid, $\mathrm{m} / \mathrm{s}$

$X \quad$ Non-dimensional coordinate along $x$ axis

$(x, y) \quad$ Coordinate axis, $\mathrm{m}$

$\left(x_{a}, x_{b}\right) \quad x$ location of the points at which the constructed interface intersects the actual interface

$\Delta H_{M} \quad$ Latent heat of melting, $\mathrm{J} / \mathrm{kg}$

$\Delta V \quad$ Volume of a control volume, $\mathrm{m}^{3}$

Non-dimensional numbers

$\begin{array}{ll}\text { Pr } & \text { Prandtl number } \\ \text { Ra } & \text { Rayleigh number } \\ \text { St } & \text { Stefan number }\end{array}$

Greek Symbols

$\begin{array}{ll}\alpha & \text { Thermal diffusivity, } \mathrm{m}^{2} / \mathrm{s} \\ \beta & \text { Volumetric expansion coefficient, } 1 / \mathrm{K} \\ \Gamma(x, y) & \text { Equation of the interface } \\ \theta & \text { Non-dimensional temperature } \\ \theta_{a}, \theta_{b} & \text { Non-dimensional temperatures at the start } \\ & \text { and end of a wavy cycle respectively } \\ \lambda & \text { A constant appearing in equation (55) } \\ \mu & \text { Dynamic viscosity, Pa-s } \\ \nu & \text { Kinematic viscosity, } \mathrm{m}^{2} / \mathrm{s} \\ \xi & \text { A coordinate along the line joining the } \\ & \text { centroids of the control volumes, } \mathrm{m} \\ \tau & \text { Density, kg/m } \\ \tau_{a}, \tau_{b} & \text { Non-dimensional time } \\ \tau^{*} & \text { Non-dimensional times at the start and end } \\ & \text { of a wavy cycle respectively } \\ \tau^{* *} & \text { Non-dimensional time at which the interface } \\ & \text { is at the centroid of the control volume } \\ & \text { Non-dimensional time when the one dimension } \\ \Phi & \text { melting system reaches pseudo steady } \\ \Omega & \text { state as predicted by classical enthalpy method } \\ & \text { A generic transport variable }\end{array}$

Subscripts

$E, W, N, S$ Tags used to address the neighbours of chosen control volume

$e, w, n, s \quad$ Tags used to address the faces of chosen control volume 


$\begin{array}{ll}f & \text { Face } \\ I & \text { Interface } \\ n b & \text { Neighbours } \\ P & \text { A tag used to address a chosen control volume } \\ n & \text { Normal } \\ x, y, \xi & \text { Partial derivatives along the corresponding directions } \\ S & \text { Solid } \\ L & \text { Liquid }\end{array}$

\section{Subscripts}

$\begin{array}{ll}S & \text { Solid } \\ L & \text { Liquid }\end{array}$

Abbreviations

CV Control Volume

SL Solid-liquid

\section{References}

Brent A D, Voller V R and Reid K J 1988 Enthalpy-porosity technique for modelling convection-diffusion phase change: Application to the melting of a pure metal. Numer. Heat Transfer 13(3): 297-318

Cao Y and Faghri A 1990 Numerical analysis of phase-change problems including natural convection. J. Heat Trans. 112(3): 812-816

Cerimele M M, Mansutti D and Pistella F 2002 Numerical modelling of liquid/solid phase transitions analysis of a gallium melting test. Comput. Fluids 31(4-7): 437-451

Crank J 1984 Free and moving boundary problems (Oxford: Oxford University Press)

Date A W 1994 A novel enthalpy formulation for multidimensional solidification and melting of a pure substance. Sadhana 19(5): 833-850

Donea J, Giuliani S and Halleux J P 1982 An arbitrary Lagrangian-Eulerian finite element method for transient dynamic fluid-structure interactions. Comput. Method Appl. M. 33(1-3): 689-723

Gartling D K 1980 Finite element analysis of convective heat transfer problems with change of phase. Computer Methods in Fluids, Morgan K, Taylor C, Brebbia C A (eds), Pentech, London, 257-284

Gua C and Viskanta R 1986 Melting and solidification of a pure metal on a vertical wall. J. Heat Trans. 108(1): 174-181

Kim S and Anghaie S 2001 An effective conduction length model in the enthalpy formulation for the Stefan problem. Int. Commun. Heat Mass 28(6): 733-741

Kumar V, Durst F and Ray S 2006 Modeling moving-boundary problems of solidification and melting adopting an arbitrary Lagrangian-Eulerian approach. Numer. Heat Tr. B-Fund. 49(4): 299-331

Lacroix M 1989 Computation of heat transfer during melting of a pure substance from an isothermal wall. Numer. Heat Tr. B- Fund. 15(2): 191-210

Li C Y, Garimella S V and Simpson J E 2003 Fixed-grid front-tracking algorithm for solidification problems, part I: Method and validation. Numer. Heat Tr. B- Fund. 43(2): 117-141

Mathur S R and Murthy J Y 1997 A pressure-based method for unstructured meshes. Numer. Heat Tr. BFund. 31(2): 195-215 
Niranjan G N and Iyer K 2011 Modified enthalpy method for the simulation of melting and solidification. Proc. $21^{\text {st }}$ National and $10^{\text {th }}$ ISHMT-ASME Heat and Mass Transfer Conf., Chennai, India, December 27-30. Paper number ISHMT_16_017

Okada M 1984 Analysis of heat transfer during melting from a vertical wall. Int. J. Heat Mass Tran. 27(11): 2057-2066

Patankar S V 1980 Numerical Heat Transfer and Fluid Flow (Washington, DC: Taylor and Francis)

Paterson S 1952 Propagation of a boundary of fusion. Glasgow Math. J. 1(1): 42-47

Pilliod Jr J E and Puckett E G 2004 Second-order accurate volume-of-fluid algorithms for tracking material interfaces. J. Comput. Phys. 199(2): 465-502

Price P H and Slack M R 1954 The effect of latent heat on numerical solutions of the heat flow equation. Br. J. Appl. Phys. 5(11): 285-287

Rhie C M and Chow W L 1983 Numerical study of the turbulent flow past an airfoil with trailing edge separation. AIAA J. 21(11): 1525-1532

Rider W J and Kothe D B 1998 Reconstructing volume tracking. J. Comput. Phys. 141(2): 112-152

Shmueli H, Ziskind G and Letan R 2010 Melting in a vertical cylindrical tube: Numerical investigation and comparison with experiments. Int. J. Heat Mass Trans. 53(19-20): 4082-4091

Tacke K 1985 Discretization of the explicit enthalpy method for planar phase change. Int. J. Numer. Meth. Eng. 21(3): 543-554

Udaykumar H S, Shyy W and Rao M M 1996 ELAFINT: A mixed Eulerian-Lagrangian method for fluid flows with complex and moving boundaries. Int. J. Numer. Meth. Fl. 22(8): 691-712

Voller V and Cross M 1981 Accurate solutions of moving boundary problems using the enthalpy method. Int. J. Heat Mass Tran. 24(3): 545-556

Voller V R, Cross M and Markatos N C 1987 Enthalpy method for convection/diffusion phase change. Int. J. Numer. Meth. Eng. 24(1): 271-284 